\title{
Mapping functional specificity in the Dfd and Ubx homeo domains
}

\author{
Lin Lin and William McGinnis \\ Department of Molecular Biophysics and Biochemistry, Yale University, New Haven, Connecticut 06511 USA
}

To define homeo domain subregions that are important for embryonic targeting specificity of homeotic proteins, we generated a series of Deformed/Ultrabithorax chimeric genes in which parts of the Deformed homeo box region were substituted with Ultrabithorax sequences. Chimeric coding regions were attached to heat shock promoters and introduced into the Drosophila genome by P-element transformation. After heat-induced ectopic expression in embryos, we examined the cuticular phenotypes induced by the resulting chimeric proteins. We also tested the ability of the chimeric proteins to regulate transcription units that are normal targets of Deformed and Ultrabithorax. Our results indicate that specific amino acid residues at the amino end of the Ultrabithorax homeo domain are required to specifically regulate Antennapedia transcription; and in the context of a Deformed protein, these amino-end residues are sufficient to switch from Deformed- to Ultrabithorax-like targeting specificity. Although residues in the amino end of the homeo domain are also important in determining a Deformed-like targeting specificity, other regions of the Deformed homeo domain are also required for full activity.

[Key Words: Homeotic genes; homeo domain; $D f d ; U b x$ ]

Received December 30, 1991; revised version accepted March 3, 1992.

The family of homeotic selector genes residing in the Antennapedia and Bithorax complexes (ANT-C and BX$\mathrm{C}$, collectively referred to as the Homeotic complex or HOM-C), determine segmental identities during development of Drosophila melanogaster (Garcia-Bellido 1977; Lewis 1978; Struhl 1982; Kaufman et al. 1990|. A dramatic demonstration of their functions is obtained from ectopic expression assays (Schneuwly et al. 1987; Gibson and Gehring 1988; Kuziora and McGinnis 1988; González-Reyes and Morata 1990; Mann and Hogness 1990), when homeotic selector proteins can induce the development of structures normally derived from one anterior-posterior axial position in ectopic positions (homeotic transformations). Sequence comparisons reveal that the proteins encoded by HOM-C selector genes conserve similar 60-amino-acid homeo domains with 60$90 \%$ sequence identity and have little sequence identity in regions outside the homeo domain (for review, see Gehring 1987; Scott et al. 1989). When tested in vitro, in tissue culture cells, in yeast cells, and in Drosophila embryos, homeo domain proteins can function as transcription activators or as repressors (for review, see Levine and Hoey 1988, Hayashi and Scott 1990), so the HOM-C proteins presumably exert their axial identity functions by transcriptional regulation of distinct sets of downstream genes.

How do the HOM-C proteins, which are all quite similar in homeo domain structure, achieve their diverse regulatory effects? Embryonic tests of mutant or chimeric versions of the Deformed (Dfd), Sex combs re- duced (Scr), Antennapedia (Antp), Ultrabithorax (Ubx), and Abdominal-B (Abd-B) homeotic proteins have indicated that residues within or immediately flanking the homeo domain are responsible for the gross distinctions in their functional specificities (Kuziora and McGinnis 1989, 1991; Gibson et al. 1990; Mann and Hogness 1990). At present, there is no biochemical explanation for the homeo domain-dependent regulatory differences between HOM-C proteins. It seems likely that differences in DNA-binding affinities and protein-protein interactions both make some contribution (Hayashi and Scott 1990; Dessain et al. 1992; Ekker et al. 1991), but the subregions of HOM-C homeo domains that confer specific DNA-protein or protein-protein contacts that are relevant in vivo are unknown.

There is much useful information concerning HOM-C homeo domain DNA-binding interactions. The homeo domain region of HOM-C proteins apparently contributes most or all of their DNA-binding activity (Müller et al. 1988; Affolter et al. 1990; Ekker et al. 1991). This is in contrast to some other homeo domain subclasses, for example, paired and POU type, where the homeo domain regions contribute only a portion of DNA-binding activity of the entire protein (Sturm and Herr 1988; Kristie and Sharp 1990; Treisman et al. 1991). Structural studies of the Antp, engrailed, and MAT $\alpha 2$ homeo domains indicate that all fold into a triple helical domain in which the second and third $\alpha$-helices are very similar in structure to the helix-turn-helix (HTH) motif of prokaryotic DNA-binding proteins (Laughon and Scott 1984; Otting 
et al. 1988; Qian et al. 1989; Wolberger et al. 1991). Homeo domain/DNA cocrystals show that residues in the third $\alpha$-helix interact with bases in the major groove of binding sites, and amino-terminal residues interact with bases in the minor groove, with many other residues making backbone contacts (Kissinger et al. 1990; Otting et al. 1990; Wolberger et al. 1991). In some homeo domains, residue 50 , which maps in the third $\alpha$-helix, has been singled out as a crucial determinant for DNA-binding specificity in vitro and in yeast cells /Hanes and Brent 1989, 1991; Treisman et al. 1989), but this position is occupied by glutamine in all HOM-C homeo domains (Fig. 1). Besides residues in the third helix, deletion and domain swap experiments have indicated that the amino-terminal end of the homeo domain is required for high-affinity interactions with DNA (Percival-Smith et al. 1990; Damante and Di Lauro 1991).

Our assays for mapping functionally important differences within the homeo domain regions of different HOM-C proteins rely on the distinct auto- and crossregulatory interactions that are normally accomplished by $D f d$ and $U b x$. Dfd protein positively autoregulates the $D f d$ transcription unit in epidermal cells of the maxillary segment (Bergson and McGinnis 1990; Regulski et al. 1991), and $U b x$ represses Antp transcription in epidermal cells of parasegment 6 (posterior T3-anterior A1; Hafen et al. 1984). These distinct targeting specificities are also reflected in the activities of Dfd protein and the Dfd/Ubx-HD protein /a chimeric protein with 66 amino acids of the Ubx homeo domain region substituted for the analogous residues of Dfd) in embryos after heat shock-induced ectopic expression. Using this ectopic expression assay for homeotic regulatory function, Dfd protein specifically activates the endogenous Dfd transcription unit in epidermal cells of the thorax and abdomen but has no effect on Antp transcription (Kuziora and McGinnis 1988; Regulski et al. 1991). The Dfd/Ubx-HD protein has no regulatory effect on $D f d$ but, instead, activates the Antp transcription unit in the embryonic head (Kuziora and McGinnis 1989). Thus, the Dfd/UbxHD protein appears to have gained the targeting specificity of Ubx while retaining the ability of the Dfd protein to activate, instead of repress, transcription.

By systematic tests of a series of chimeric proteins in which homeo domain subregions were swapped, we wished to ask which subregions were most important in discriminating Dfd and Ubx homeo domain functions. By comparing the behavior of each hybrid protein with the Dfd and Dfd/Ubx-HD parental proteins, we find that the amino end of the Ubx homeo domain is both required and, to some extent, sufficient for the selective regulation of Antp.

\section{Results}

In the 66-residue homeo domain region of $\mathrm{Ubx}$ protein that was substituted to generate the Dfd/Ubx-HD chimera, there are 22 amino acid residues that are Ubx specific. Using these Ubx-specific residues and restriction sites that already were shared, or were intentionally en-
Figure 1. Dfd/Ubx homeo domain chimeras. (Top) The embryonic expression vector for the proteins containing chimeric Dfd/ Ubx homeo domains. The P-element vectors that were used contained hsp 70 upstream sequences fused to a $D f d$ cDNA and Dfd 3' sequences, including polyadenylation sites (Kuziora and McGinnis 1988). An enlarged homeo domain region is depicted below the vector, and the 66-amino-acid residues encoded by $D f d$ in this region are shown in one-letter format. The 22 positions in this region where the Ubx protein sequence differs from Dfd are also indicated. These 22 different amino acids were divided into four subregions according to their putative structural identities: the amino $(\mathrm{N})$ end, helix I, helix II/turn, and the carboxyl $(\mathrm{C})$ end. The four subregions were swapped between $D f d$ and $U b x$ using three restriction sites (AccI, MluI, and $B g / I I)$, some of which were created by sitedirected mutagenesis (Materials and methods). This resulted in six $D f d / U b x$ homeo box chimeras, designated as hsS1-hsS6, all in the context of $D f d$ cDNA sequence. The homeo domain composition of these subregion chimeras are diagramed with solid bars designating Ubx-like sequences and shaded bars showing Dfd-like sequences.

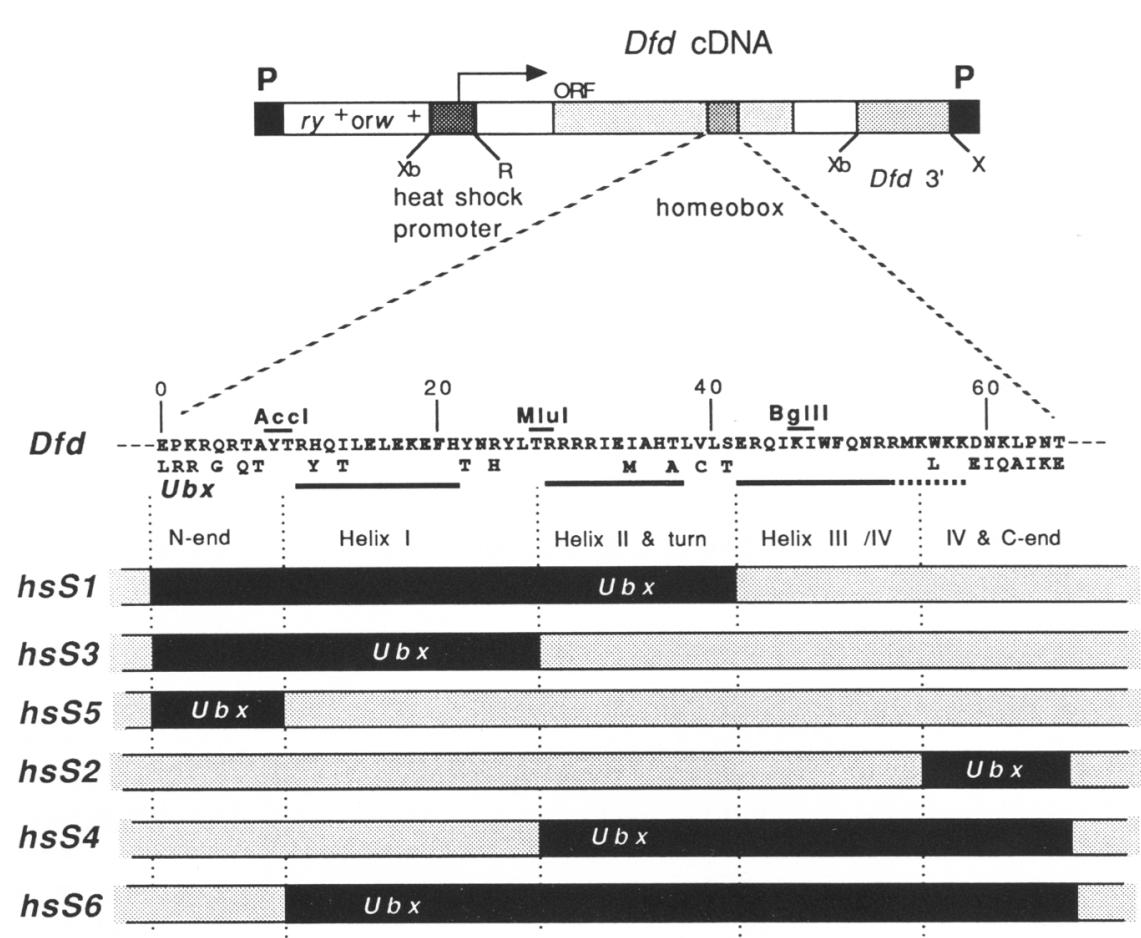


gineered (Materials and methods) into both $U b x$ and $D f d$ homeo box sequences, we could test four structural subregions of the homeo domain. As shown in Figure 1, these subregions are the amino end $(\mathrm{N}$-end; residues $0-7$ ), $\alpha$-helix I (residues 1 1-24), $\alpha$-helix II and turn region (residues 34-41), and the carboxyl end (C-end; residues 56-65). We constructed three pairs of reciprocal chimeric homeo boxes that were all introduced into a $D f d$ cDNA sequence under $h s p 70$ promoter control. The $h s p 70$ promoter/Swap 1 chimera ( $h s S 1$ ) encodes a normal Dfd protein sequence except in the amino end, helix I, and helix II/turn subregions, which encode Ubx-specific residues. The hsS3 chimera encodes Ubx-specific residues only in the amino end and helix I subregions, and the hs $S 5$ construct encodes Ubx-specific residues only in the aminoend subregion (Fig. 1). In the reciprocal set of chimeras, $h s S 2$ encodes Ubx-specific residues only in the carboxyend subregion, hsS4 encodes Ubx-specific residues in both the helix II/turn and carboxy-end subregions, and hsS6 encodes Ubx-specific residues in the helix I, helix II/turn, and carboxy-end subregions.

Multiple transgenic lines carrying genomic inserts of the hsS1-hsS6 chimeric genes were generated using P-element transformation (Rubin and Spradling 1982; Spradling and Rubin 1982), and transformant functions were tested in fly strains carrying two chimeric gene copies. The expression of chimeric proteins was induced in $h s S 1-h s S 6$ embryos with a double heat shock regimen. A 1-hr heat shock $\left(37^{\circ} \mathrm{C}\right)$ was administered at the cellular blastoderm stage, followed by a l-hr recovery at normal growth temperature $\left(25^{\circ} \mathrm{C}\right)$; a second $37^{\circ} \mathrm{C}$ heat shock of $1 \mathrm{hr}$ was then administered, after which embryos were returned to normal temperatures. To test the relative expression levels, stabilities, and cellular localizations of the chimeric proteins as compared with parental hsDfd and hsDfd/Ubx-HD proteins, all lines were stained with anti-Dfd antiserum at 1 and $3 \mathrm{hr}$ after a double heat shock regimen (data not shown). The parental and chimeric proteins were expressed at approximately equal levels as judged by staining intensity, and all were localized in embryonic nuclei. In addition, all proteins were still detectable (barely) after $3 \mathrm{hr}$ of recovery, so they appeared to have approximately the same levels of stability.

\section{Homeotic transformations induced by hsS1-hsS6 chimeras}

To test the functional specificity of the chimeric proteins, we first evaluated the cuticular phenotypes induced by the chimeric proteins after heat shock-induced expression. The parental constructs hsDfd and hsDfd/ $U b x-H D$ each induce homeotic transformations by indirect means. The ectopic and persistent autoactivated expression of Dfd protein that is induced by $h s D f d$ triggers a transformation of parts of more posterior segments to maxillary identities, as evidenced by the development of maxillary cirri and (occasionally) mouth hooks in labial and thoracic segments (Fig. 2b; Kuziora and McGinnis 1988). In $D f d$ mutant backgrounds, the $h s D f d$ construct does not induce ectopic maxillary structures. The hs$D f d / U b x-H D$ construct ectopically activates the expression of Antp transcripts and protein, which triggers a transformation of parts of more anterior segments toward thoracic identities, as evidenced by the development of thoracic denticle belts in the head (Fig. 2c; Kuziora and McGinnis 1989|. In Antp mutant backgrounds, the $h s D f d / U b x-H D$ construct does not induce ectopic thoracic structures. Thus, whether using single or double heat shock regimens, neither hsDfd nor hsDfd/Ubx$H D$ induces a strong phenotypic transformation that is directly caused by protein produced from the heat shock promoter construct, which may be due in part to the relative instability of ectopically expressed Dfd and Dfdchimeric proteins when compared with ectopically expressed Ubx or Antp proteins (Gibson and Gehring 1988; Kuziora and McGinnis 1988; González-Reyes and Morata 1990; Mann and Hogness 1990).

The hsS1-hsS6 subregion chimeras can be divided into two groups based on the cuticular phenotypes they induced. When treated with a double heat shock regimen, embryos carrying $h s S 1, h s S 3$, and $h s S 5$ chimeras had severe head involution defects and developed thoracic denticle belts in place of normal head structures. Almost all of the hsS1, hsS3, and hsS5 embryos die before hatching when heat-shocked, although the different chimeric genes yield different levels of penetrance and expressivity for the head-to-thorax transformations. hsS1 and hsS3 give strong transformations, yielding phenotypes essentially identical to the parental $h s D f d / U b x-H D$ construct $\mid \sim 90 \%$ of unhatched first-instar larvae of both transformants had ectopic denticles in the head segments; Fig. 2d,e). The hsS5 construct is a slightly weaker version of the hsDfd/Ubx-HD parent, as patches of ectopic thoracic denticles appeared in the head in $~ 70 \%$ of heat-shocked hsS5 larvae (Fig. 2f). None of these three transformants yield $h s D f d$-like homeotic transformations after heat shock.

The heat-shocked hsS2, hsS4, and hsS6 transformants did not have cuticular phenotypes that resembled either of the parental constructs. The expression of the hsS2 construct appeared to be rather innocuous, as almost $90 \%$ of hsS2 embryos survived the heat shock treatment with no obvious ill effect. The remaining unhatched $h s S 2$ larvae exhibited only defects in head involution (Fig. 2g). Heat shock-induced expression of hsS4 and hsS6 constructs in embryos had stronger effects on embryonic viability, as only $40 \%$ of embryos hatched. The $h s S 4$ and hsS6 animals that died as unhatched first-instar larvae had no specific morphological changes except for abnormal head involution (Fig. $2 \mathrm{~h}, \mathrm{i}$ ). One rare exception to this is that in small percentages of hsS4 $(3 \%)$ and hsS6 $(6 \%)$ cuticles, there were a few randomly positioned denticles in the head regions.

\section{Transcriptional regulatory effects of the hsS1-hsS6 chimeras}

As mentioned previously, the parental constructs have distinct embryonic targeting specificities, with $h s D f d$ 

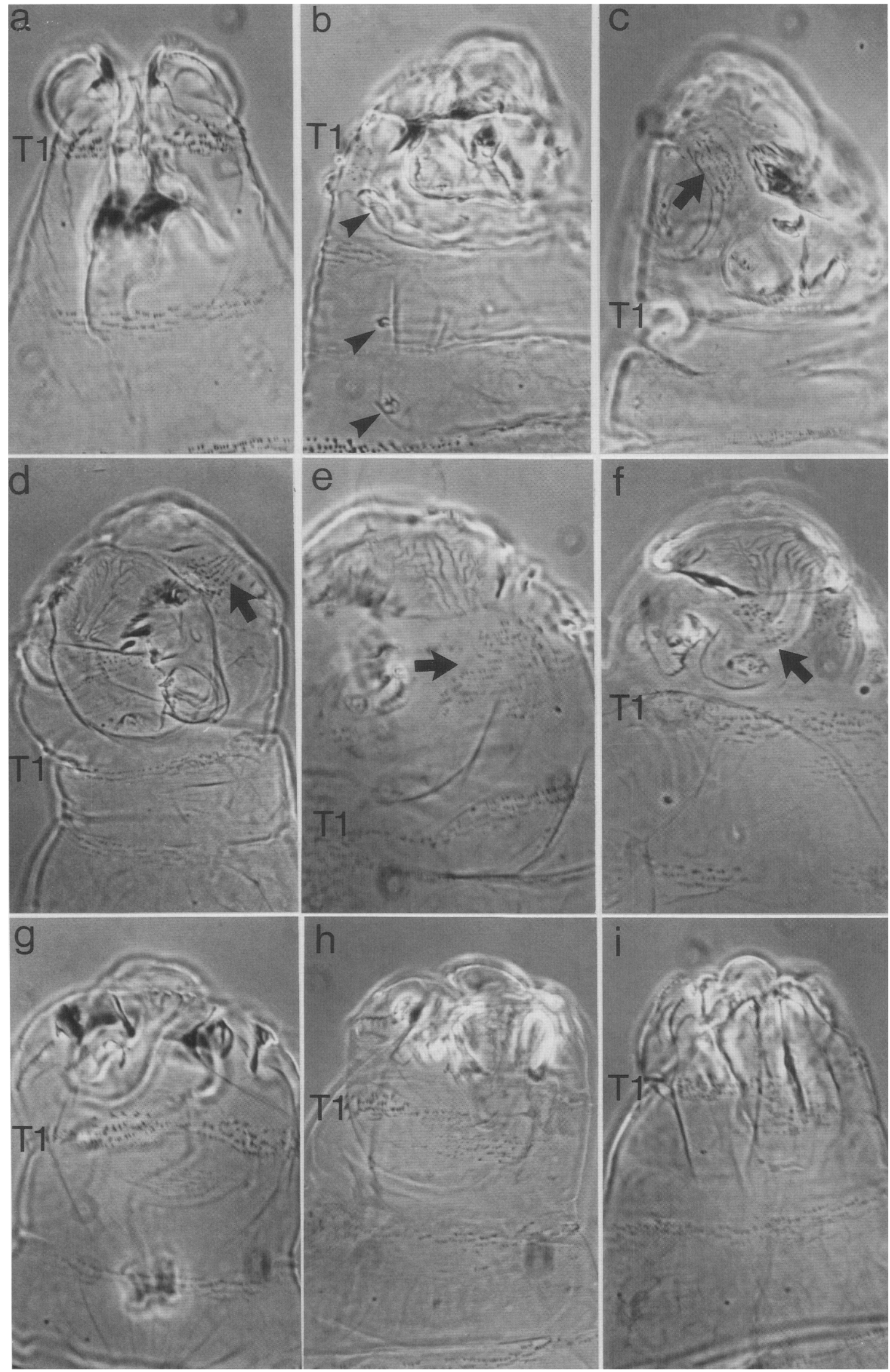

Figure 2. (See facing page for legend.) 
activating ectopic $D f d$ transcription (Fig. $3 \mathrm{c}$ ), and $h s D f d$ / $U b x-H D$ activating ectopic Antp transcription (Fig. 3f). To test the targeting specificity of the hsS1-hsS6 chimeras, digoxigenin-labeled $D f d$ and Antp cDNA probes were used in whole-mount in situ hybridizations on heat-shocked embryos. As shown in Figure 3, hsS1, hsS3, and $h s S 5$ activate ectopic Antp transcription. Qualitatively, $h s S 1$ and $h s S 3$ appear to have stronger regulatory effects, variably activating Antp transcription in the first thoracic, labial, and head epidermis (Fig. 3h,j). These patterns of ectopic Antp transcription closely resemble those induced by $h s D f d / U b x-H D$ (Fig. $3 f$ ). The $h s S 5$ construct also induces ectopic Antp transcripts, but appears to act in fewer cells overall (Fig. $3 \mathrm{~m}$ ). We have detected no significant effects on Antp expression levels within the normal Antp expression domain. However, the abundance and pattern of Antp transcripts evolves rapidly during development, so modest differences from the normal patterns would be difficult to detect.

None of the three constructs activate ectopic $D f d$ transcription in any epidermal cells in a manner similar to the hsDfd parent (Fig. 3c,g,i,k). However, the level of normal $D f d$ expression in the maxillary and mandibular segments is repressed in almost all the hsS 3 embryos after heat shock (Fig. 3i).

In heat-shocked $h s S 2$, hsS4, and hsS6 embryos, the distribution of Antp transcripts closely resembles the normal pattern: No ectopic expression is detected (Fig. $4 \mathrm{~b}, \mathrm{~d}, \mathrm{f})$; nor are the levels or patterns of Antp transcription obviously affected within the normal Antp expression domain, though again, modest differences from the normal patterns would be difficult to detect.

The hsS2 and hsS4 constructs induce weak regulatory effects on $D f d$ transcription that do not resemble those induced by the parental hsDfd construct. About $45 \%$ of hs $S 2$ embryos accumulate $D f d$ transcripts in a few cells of the most anterior segment, the clypeolabrum (Fig. 4a), whereas the rest of the hsS2 embryos display a normal $D f d$ expression pattern. Ectopic $D f d$ expression in the clypeolabrum has not been observed in $h s D f d$ embryos (Fig. 3c). The only detectable effect of hsS4 expression is a repression of $D f d$ transcript levels within the normal expression domain of the maxillary and mandibular segments (Fig. 4c). In $74 \%$ of hsS6 embryos, ectopic Dfd transcripts are detected in a few cells of the labial segment, which weakly resembles the $h s D f d$ regulatory effect (Fig. 4e). hsS6 also induces $D f d$ transcription in a few cells of the clypeolabrum.

\section{Discussion}

The data from both the cuticular and transcriptional regulatory assays of hsS1-hsS6 is summarized in Figure 5, in which each construct is compared with the parental $h s D f d$ and $h s D f d / U b x-H D$. The most important conclusion from these data is that only a few amino-end Ubxspecific homeo domain residues are required to confer targeting specificity for the regulation of Antp transcription. $h s S 1, h s S 3$, and $h s S 5$ all retain the specificity of the parental $h s D f d / U b x-H D$ construct, and the protein encoded by $h s S 5$ only differs from Dfd at $6 \mathrm{Ubx}$-specific residues at the amino end of the homeo domain (Fig. 6). The same region is also necessary for Ubx-like targeting, as hsS6 is incapable of regulating Antp even though the hsS6 homeo domain is entirely Ubx-like except at the amino end.

A second conclusion is that both amino- and carboxyend Dfd-specific homeo domain residues are required for full ectopic activation of the endogenous $D f d$ transcription unit, although the amino end is sufficient for a weak Dfd-like regulatory effect. As can be seen in hsS2 and hsS5 (Fig. 5), changing either amino or carboxyl ends to Ubx-specific residues abolishes the ability to activate $D f d$ transcription. Only the $h s S 6$ chimera had a detectable, although weak, ability to activate $D f d$ transcription in a way similar to $h s D f d$, again emphasizing the importance of the homeo domain amino end. However, it is puzzling that the hsS2 and hsS4 proteins, with even more Dfd-like homeo domain residues than hsS6, were incapable of activating $D f d$ transcription.

Curiously, the hsS3 and hsS4 chimeras that are halfDfd/half-Ubx (recombined in the helix I/II region) both appear to partially repress $D f d$ transcription. This result is puzzling because the hsS3 and hsS4 swaps are nonoverlapping and also because the regulatory effect on $D f d$ does not resemble either of the parental constructs. This seems to suggest that an intact helix I and II region (either Ubx- or Dfd-specific) is required to prevent the Dfd or Ubx homeo domains from mediating a repressive effect on $D f d$ transcription. None of the other chimeric proteins have a detectable repressive effect on $D f d$ transcription, so it seems possible that the generation of chimeric helix I/II regions in hsS3 and hsS4 supplies novel functions not present in either of the parental constructs.

Finally, we note that there is a correlation between abundant ectopic activation of an endogenous target

Figure 2. Cuticular phenotypes induced by hsS1-hsS6 proteins in embryos. Experimental (hsS1-hsS6) and control (hsDfd, hsDfd/ $U b x-H D$, and $r y^{506}$ or $w^{1006}$ host strains/ embryos were heat-shocked for $1 \mathrm{hr}$ at cellular blastoderm stage, recovered for $1 \mathrm{hr}$, and heat shocked for $1 \mathrm{hr}$. The resulting first-instar larvae were scored for survival and for cuticular defects and segmental transformations (Van der Meer 1977). The first thoracic segment of each larvae is marked by Tl. (a) ry ${ }^{506}$ (P-element host strain) larva (note the normal, fully involuted head). (b) hsDfd larva: Ectopic maxillary cirri in labial and thoracic segments are shown by arrowheads. $(c) h s D f d / U b x-H D$ larva: Strong homeotic transformation of head to thorax is shown by T2-like denticle belts in the head (arrow). $(d-f)$ hsS1, hsS3, and hsS5 larvae, respectively. All had head-to-thoracic transformations, similar to those seen in $h s D f d / U b x-H D$ larvae as shown in $c$. Arrows indicate the ectopic thoracic-like denticles in the head region. $(g-i)$ The $h s S 2$, $h s S 4$, and hsS6 larval cuticles. No reproducible homeotic transformations were observed, although some larvae, as shown, had moderate head involution defects. 


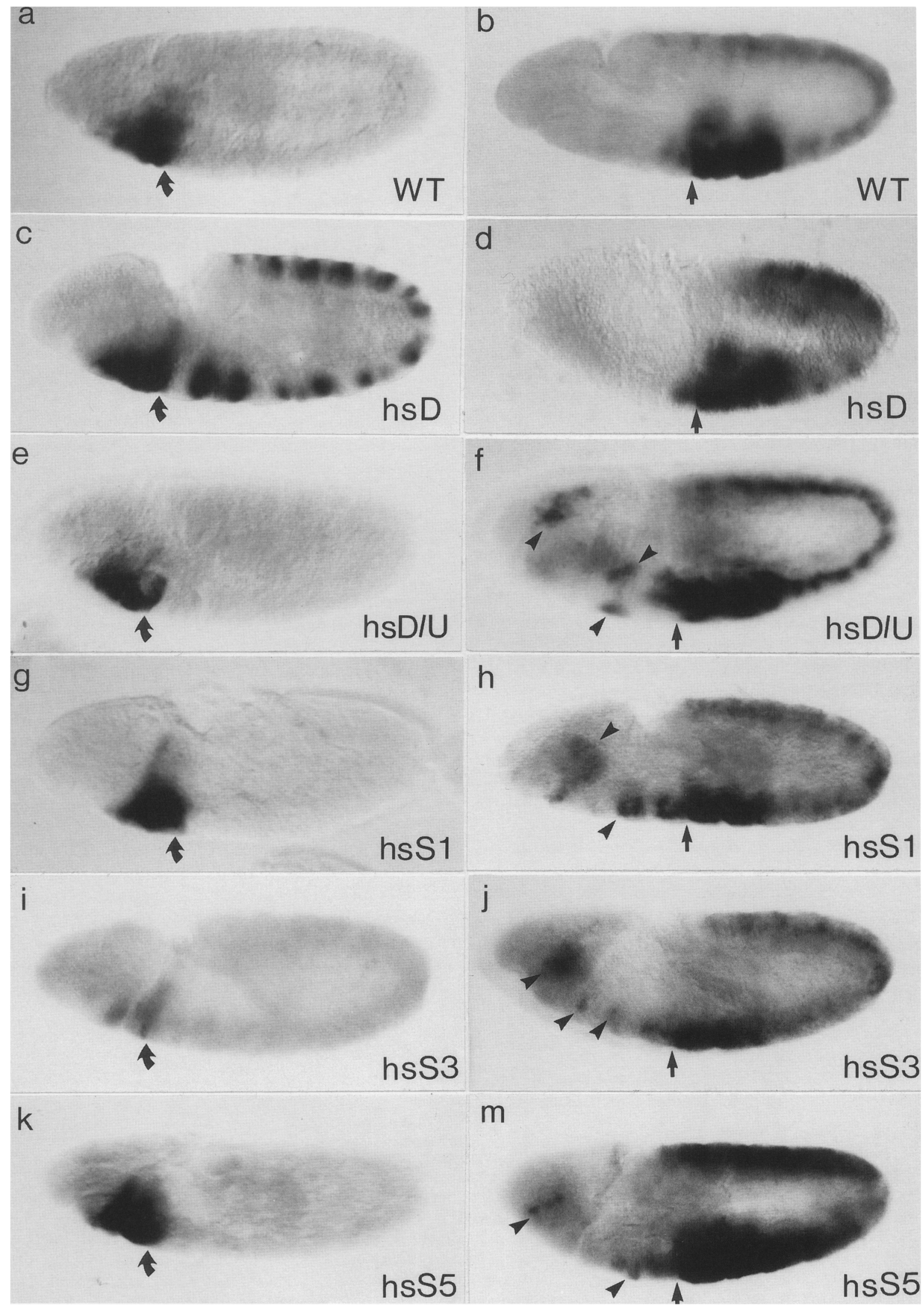

Figure 3. (See facing page for legend.) 
gene (Dfd or Antp) and the induction of a cuticular homeotic transformation (Fig. 5), consistent with all of the cuticular phenotypes deriving from ectopic expression of endogenous homeotic genes. Head-to-thorax transformations were only detected in hsS1, hsS3, and hsS5 strains, all cases in which endogenous Antp was ectopically expressed at abundant levels in the embryonic head. Unlike the $h s D f d$ parental construct, none of the subregion chimeras induced abundant ectopic expression of the endogenous $D f d$ gene, and no ectopic maxillary structures were reproducibly seen in the $h s S 1-h s S 6$ strains. This is in agreement with previous experiments on $h s D f d$ and $h s D f d / U b x-H D$ constructs, indicating that their homeotic effects were reverted in $D f d$ and Antp mutant backgrounds, respectively (Kuziora and McGinnis 1988, 1989).

Previous experiments have shown that Dfd proteinbinding sites are required for the function of an autoregulatory element module that supplies late expression in maxillary epidermal cells (Bergson and McGinnis 1990; Regulski et al. 1991). Thus, the action of $h s D f d$ is likely to be accomplished by direct binding of Dfd protein to sites in the upstream autoregulatory element of the endogenous $D f d$ gene (Regulski et al. 1991). Our working model is that Dfd/Ubx-HD protein and the chimeric proteins that mimic its function (hsSl, hsS3, and hsS5) also act directly on Antp cis-regulatory sequences (Beachy et al. 1988), but no strong evidence exists at present to support this idea. Regulatory regions that mediate the effect of Ubx on Antp in embryos have not yet been defined precisely, although recent experiments suggest that sequences in the Antp P1 first exon are required for the repressive effects of Ubx as well as for the activating effects of Dfd/Ubx-HD proteins (Zink et al. 1991; L. Lin and Y. Engstrom, unpubl.).

At present, the biochemical interactions that explain how HOM-C homeo domain proteins sort out target regulatory elements are poorly understood. Some HOM-C homeo domain proteins show modest distinctions in their preference for different binding sites (Affolter et al. 1990; Ekker et al. 1991; Florence et al. 1991; Dessain et al. 1992), but it is unclear whether these modest binding differences are important in distinguishing embryonic target elements. It seems likely, on the basis of analogy to yeast mating-type homeo domain proteins al and $\alpha 2$, and POU-class homeo domain proteins like Oct-1, Oct2, and I-POU (Keleher et al. 1988; Jarvis et al. 1989; Stern et al. 1989; Ingraham et al. 1990; Treacy et al. 1991), that stable protein-protein interactions involving different homeo domain and as yet unidentified cofactors may make an important contribution to HOM-C targeting specificity (Hayashi and Scott 1990).

Our results indicate that the amino-terminal residues are very important for both Ubx and Dfd homeo domain specificity in embryos but give no hint as to mechanism. Nevertheless, it is interesting to consider our results on the importance of different homeo domain subregions in light of recent structural descriptions of homeo domainDNA complexes (Kissinger et al. 1990; Otting et al. 1990; Wolberger et al. 1991). An intriguing feature of the homeo domain-DNA complexes is the interactions between residues in an amino-terminal "arm" and bases in the minor groove. In the engrailed homeo domain, Arg-3 and Arg-5 (Fig. 6) interact directly with A-T base pairs at the $5^{\prime}$ end of a TAAT core sequence. However, differences in positions 3 and 5 could not account for the amino-terminal targeting differences documented here as the Dfd and Ubx homeo domains have Arg at both positions.

It seems plausible that other amino acid differences in the amino-terminal arm might directly or indirectly control differences in DNA-binding affinity, which might explain the functional differences that distinguish the hsS5 and Dfd proteins. However, we have not yet been able to detect such DNA-binding differences using in vitro assays. Using monomer-binding sites with high affinity for either Ubx or Dfd homeo domains (Dessain et al. 1992; Ekker et al. 1991; Regulski et al. 1991), we have compared hsS1-hsS6 protein binding to the parental Dfd and Dfd/Ubx-HD proteins. The in vitro binding preferences of hsSl-hsS6 proteins for high-affinity Ubx or Dfd monomer-binding sites show no correlation with the tar-

Figure 3. Regulatory effects on $D f d$ and Antp transcription in $h s S 1, h s S 3$, and hsS5 embryos. Experimental $(h s S 1, h s S 3, h s S 5)$ or control $(h s D f d, h s D f d / U b x-H D)$ embryos were heat-shocked at cellular blastoderm stage for $1 \mathrm{hr}$ at $37^{\circ} \mathrm{C}$, allowed to recover for 1 hr at $25^{\circ} \mathrm{C}$, and heat-shocked again for $1 \mathrm{hr}$. After another hour at $25^{\circ} \mathrm{C}$, embryos were fixed and Dfd and Antp transcripts were detected by whole-mount in situ hybridization (Materials and methods). The different strains tested are indicated at the bottom right corner of each panel. Embryos shown in the left column were hybridized with $D f d$ probe. The curved arrows indicate the border between maxillary and labial segment in each embryo. (a) A wild-type (wt) embryo showing the normal Dfd transcript expression pattern. (c) $h s D f d$ embryos exhibit ectopic $D f d$ transcripts in labial, thoracic and abdominal segments. These $D f d$ transcripts derive from the endogenous $D f d$ locus, as the $D f d$ or $D f d / U b x$ chimeric transcripts that arise from the heat shock promoter are very unstable, with a half-life on the order of a few minutes (Kuziora and McGinnis 1988; M. Kuziora, unpubl.). (e) hsDfd/Ubx-HD embryos Dfd expression was normal. $\{g, i, k\}$ No $D f d$ transcription was ectopically activated in $h s S 1$, $h s S 3$, and $h s S 5$ embryos. However, in nearly all of the hsS3 embryos $\{i\}, D f d$ expression was repressed in the maxillary and mandibular regions. Embryos shown in the right column were hybridized with Antp probe. Arrows indicate the parasegment 3/4 boundary in each embryo, and arrowheads indicate sites of ectopic Antp transcription. (b) A wild-type (wt) embryo showing the normal Antp transcript expression pattern. (d) hsDfd embryos have normal Antp transcription patterns. $(f)$ hsDfd/Ubx-HD embryos have ectopic Antp transcripts in thoracic, labial, and head segments. $(h, j, m)$ In $h s S 1, h s S 3$, and $h s S 5$ embryos, ectopic Antp transcripts were expressed in thoracic, labial, and head segments, similar to the pattern in $h s D f d / U b x-H D$ embryos. $h s S 5$ embryos $(\mathrm{m})$ generally have fewer cells expressing ectopic Antp transcripts. All transformant strains in this figure and in Fig. 4 were heat shocked in parallel with control strains, and hybridized and stained for the same intervals, with aliquots of the same probes and solutions. 


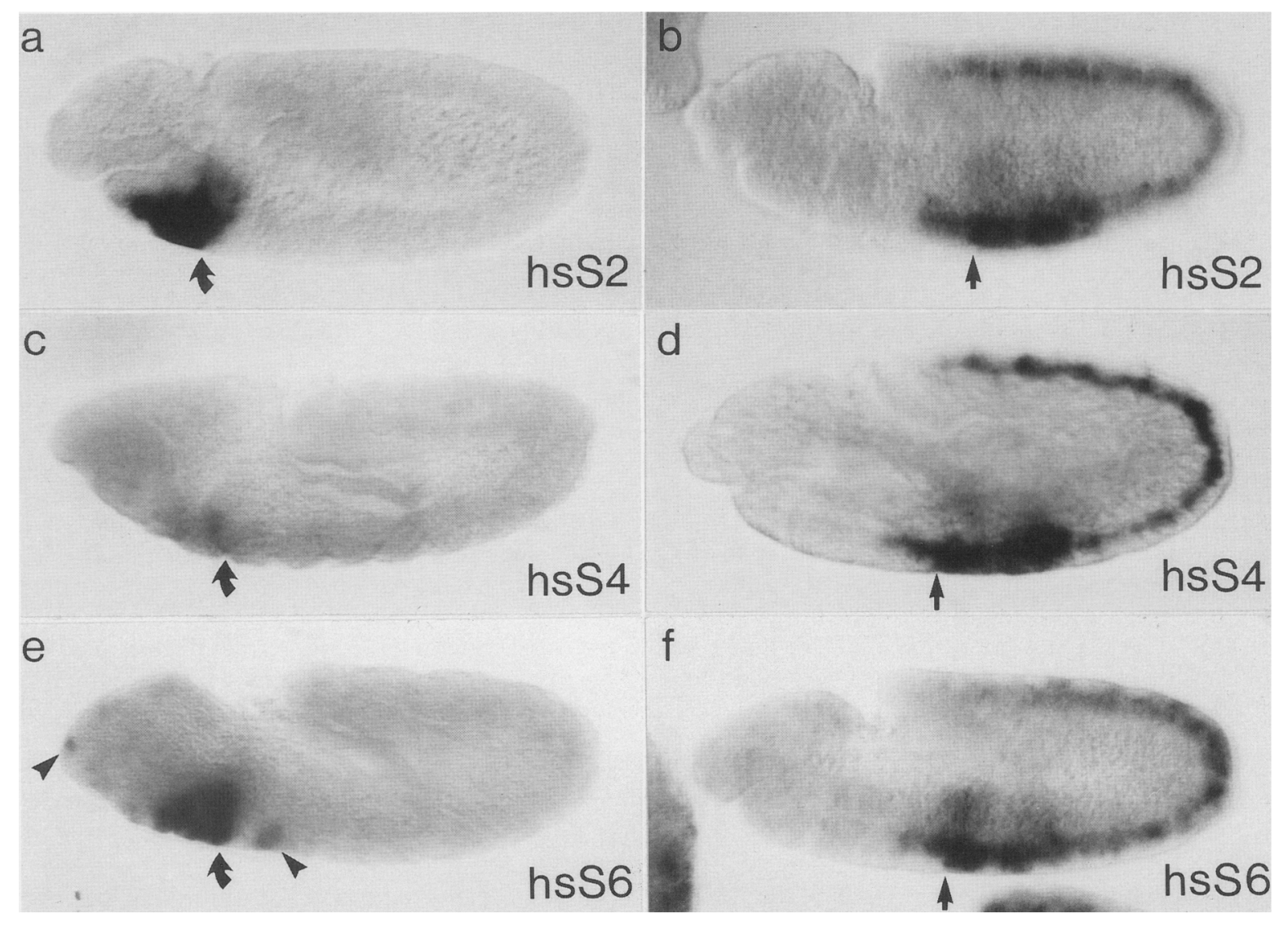

Figure 4. Regulatory effects on Dfd and Antp transcription in hsS2, hsS4, and hsS6 embryos. Experimental (hsS2, hsS4, hsS6) or control (hsDfd, hsDfd/Ubx-HD) embryos (shown in Fig. $3 \mathrm{c}-\mathrm{f}$ ) were heat-shocked at cellular blastoderm stage for $1 \mathrm{hr}$ at $37^{\circ} \mathrm{C}$, allowed to recover for $1 \mathrm{hr}$ at $25^{\circ} \mathrm{C}$, and heat shocked again for $1 \mathrm{hr}$. After another hr at $25^{\circ} \mathrm{C}$, embryos were fixed, and Dfd and Antp transcripts were detected by whole-mount in situ hybridization (Materials and methods). The different strains tested are indicated at bottom right corner of each panel. Embryos shown in the left column were hybridized with $D f d$ probe. The curved arrows indicate the border between maxillary and labial segment in each embryo; arrowheads indicate sites of ectopic $D f d$ transcription. No significant $D f d$ ectopic expression was detected in $h s S 2(a)$ and $h s S 6(e)$ embryos, as compared with hsDfd (Fig. 3c), except for a few cells of the clypeolabrum in some $h s S 2$ and $h s S 6$ embryos, and a few cells of the labial segment in hsS6 embryos. In hsS4 embryos (c), Dfd transcript levels in mandibular and maxillary segments were repressed. Embryos shown in the right column were hybridized with Antp probe. Arrows indicate the parasegment $3 / 4$ boundary in each embryo. Antp transcript patterns were normal in $h s S 2(b)$, $h s S 4$ $(d)$, and $h s S 6(f)$ embryos compared with wild-type embryos at comparable stages.

geting specificities that they exhibit in embryos (Fig. 5). For example, the hsS5 protein does not preferentially bind to monomer Ubx-binding sites in a way that closely resembles the parental Dfd/Ubx-HD (C. Gross, unpubl.).

Perhaps the different side chains in the amino-terminal region, many of which are directed away from the DNA in the homeo domain-DNA stuctures of Kissinger et al. (1990) and Wolberger et al. (1991), control required interactions with homeo domain cofactors. Specific amino-terminal residues of the homeo domain region of the Drosophila I-POU protein have been shown to be important in conferring its ability to form heteromeric complexes with the Cfl-a protein (another protein of the POU-homeo domain class; Treacy et al. 1991). These observations suggest that the mechanistic explanation for the ability of the S5 protein to specifically regulate
Antp may reside in its ability to interact productively with normal Ubx cofactors.

\section{Materials and methods}

Site-directed mutagenesis and construction of chimeric genes

Three natural or engineered restriction sites were used to swap the four subregions between the $U b x$ and $D f d$ homeo boxes (the positions of the restriction sites are shown in Fig. 1). BglII sites are present in both wild-type homeo box sequences, whereas an MluI site is present only in the $D f d$ homeo box. Using sitespecific mutagenesis (Kunkel 1985), we introduced AccI sites into both homeo boxes and an MluI site into the Ubx homeo box without changing the amino acid code. Both MluI and AccI sites were engineered in the BamHI-EcoRV fragment of the $D f d /$ Ubx chimeric gene (Kuziora and McGinnis 1989) in MP18 


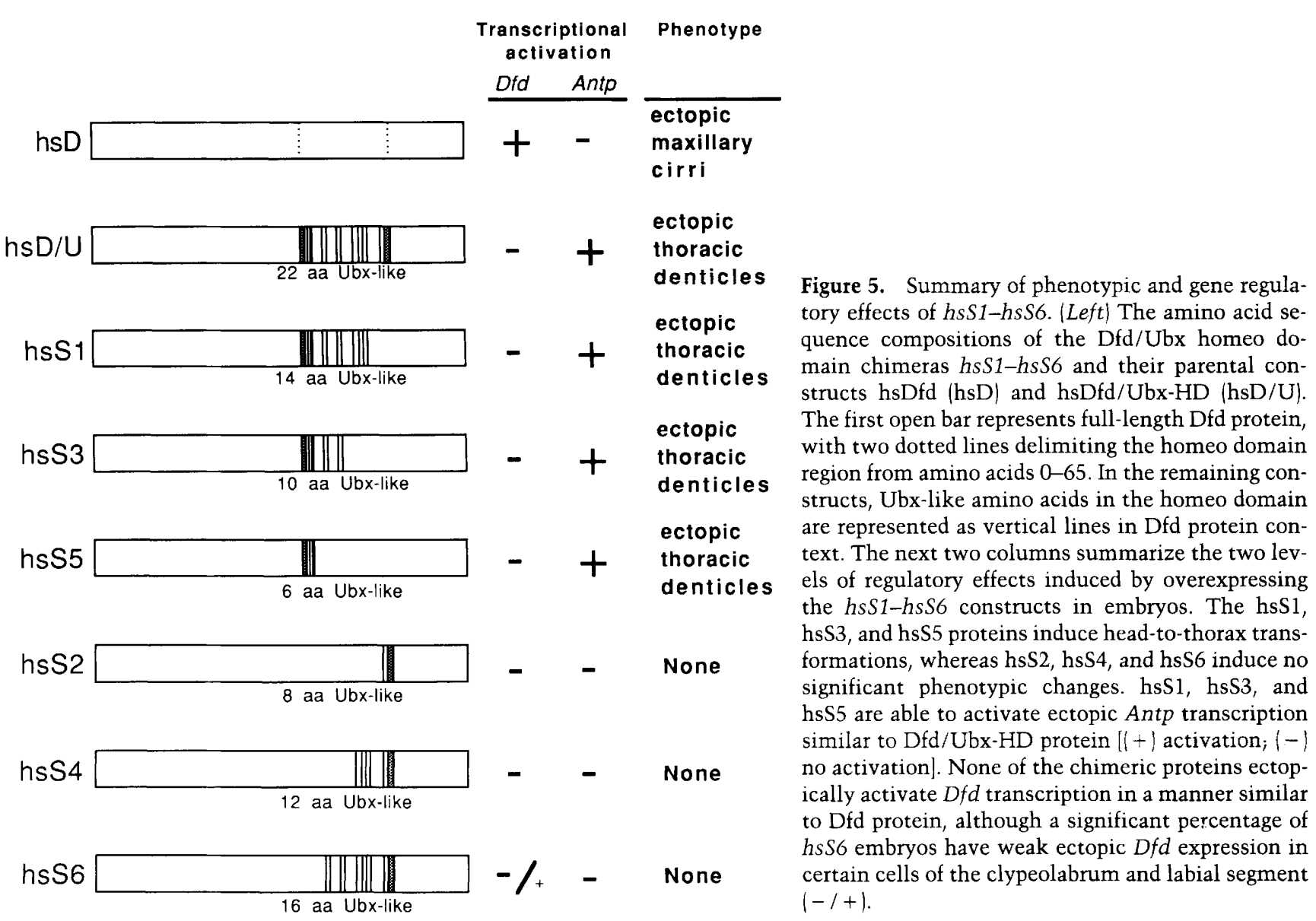

(MP18DU) by using two 24-mer oligonucleotides (MP18DU-M and MP18DU-A), whereas an $A c c$ I site was created in the BamHI-EcoRV fragment of the $D f d$ gene in MP18 (MP18D) by using a 48-nucleotide oligomer (MP18D-A). The DNA sequences of all chimeras were determined to ensure that no nonspecific mutations were introduced.

PhsS1 and PhsS2 We began with two plasmid clones made by Kuziora and McGinnis (1988, 1989): phsDfd, which contained a full-length $D f d$ cDNA under the control of the $h s p 70$ promoter, and pMK84, which was identical to phsDfd except that the $D f d$ homeo box sequence was substituted with $U b x$ homeo box sequences from codon 0 to 65 . The BglII-EcoRV fragments from phsDfd and pMK84 were exchanged, generating two recombinant plasmids from which the NotI-Sall fragments were subsequently cloned into pDM30, the Carnegie 20-based injection vector (Mismer and Rubin 1987).

PhsS3 and PhsS4 Three-fragment ligations were carried out with the following fragments: For hsS3, these included the BamHI-EcoRV fragment from phsDfd, the BamHI-MluI fragment from MP18DU-M, and the MluI-EcoRV fragment from MP18D; for hsS4, these included the BamHI-EcoRV fragment from phsDfd, the BamHI-MluI fragment from MP18D, and the

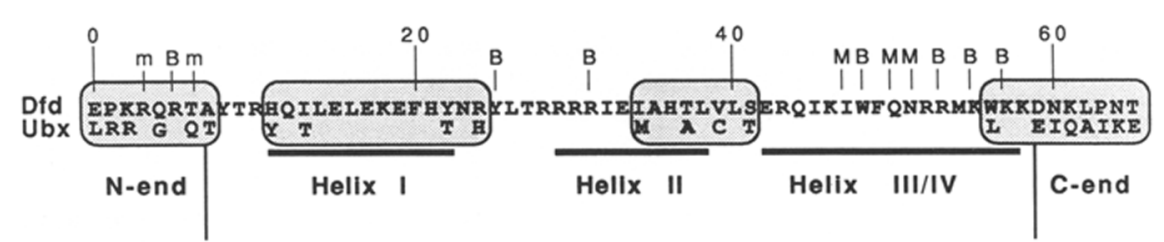

Required and sufficient for Ubx target specificity

Required for activation of $D f d$ transcription
Required for activation of $D f d$ transcription

Figure 6. Discriminator residues in the Dfd and Ubx homeo domains. The Dfd amino acid sequence in the homeo domain region is presented in the one-letter format, and residues that differ in Ubx are shown below. The 6 amino acids at the amino end of the Ubx homeo domain function as discriminator residues for Ubx homeo domain functional specificity in these embryonic assays. Both amino and carboxyl ends of the Dfd homeo domain are required for Dfd homeo domain func-

tional specificity, although the amino end appears to be more important as the $h s S 6$ construct has a weak ability to activate Dfd transcription. The symbols $(\mathrm{m}, \mathrm{M}$, and $\mathrm{B})$ above the sequence indicate positions of contacts between engrailed homeo domain residues and a DNA-binding site (Kissinger et al. 1990). (m) Minor groove base interactions; (M) major groove base interaction; (B) phosphate backbone interactions. 
MluI-EcoRV fragment from MP18DU-M. The NotI-Sall fragments of the two resulting recombinant plasmids from the three-fragment ligations were subcloned into the PstI site of the CaSpeR injection vector (Pirotta 1988) using oligonucleotide linkers.

PhsS5 and PhsS6 Three-fragment ligations were performed with the following fragments: For hsS5, these included the $B a m H I-E c o$ RV fragment from phsDfd, the BamHI-AccI fragment from MP18DU-A, and the AccI-EcoRV fragment from MP18D-A; for hsS6, these included the BamHI-EcoRV fragment from phsDfd, the BamHI-AccI fragment from MP18D-A, and the AccI-EcoRV fragment from MP18DU- A. NotI-Sall fragments of the two resulting recombinant plasmids were subcloned into the CaSpeR P-transformation vector.

\section{Transformation and functional tests of chimeric genes}

The injection procedure has been described previously (Rubin and Spradling 1982; Spradling and Rubin 1982). PhsS1 and PhsS2 constructs were coinjected with helper plasmid p $\pi 25.7$ wc (Karess and Rubin 1984) into ry ${ }^{506}$, whereas PhsS3, PhsS4, PhsS5, and PhsS6 were coinjected with $\mathrm{p} \pi 25.7 \mathrm{wc}$ into $w^{1006}$ embryos. All transformants were made homozygous, and at least two independent lines for each inserted construct were tested in our experimental assays to avoid potential problems with position effects.

For analysis of cuticular phenotypes, embryos were collected and staged to $2.5-3 \mathrm{hr}$ after egg lay (approximately cellular blastoderm/early gastrulation stages; Campos-Ortega and Hartenstein 1985), heat-shocked at $37^{\circ} \mathrm{C}$ for $1 \mathrm{hr}$, recovered for $1 \mathrm{hr}$ at $25^{\circ} \mathrm{C}$, and heat-shocked again at $37^{\circ} \mathrm{C}$ for $1 \mathrm{hr}$. The embryos were allowed to develop at $25^{\circ} \mathrm{C}$ for $\sim 36 \mathrm{hr}$. Cleared cuticular preparations were done following the procedure of Van der Meer (1977). Cuticular defects were examined and scored under a phase-contrast microscope.

For anti-Dfd protein antibody staining and whole-mount in situ hybridization, embryos at cellular blastoderm stage were heat shocked for $1 \mathrm{hr}$ at $37^{\circ} \mathrm{C}$, returned to $25^{\circ} \mathrm{C}$ for $1 \mathrm{hr}$, and heat shocked again for $1 \mathrm{hr}$. The embryos were then allowed to develop for 1 (or 3 ) more $\mathrm{hr}$ at $25^{\circ} \mathrm{C}$ before fixing and further processing. Staining with rabbit anti-Dfd protein antiserum was performed as described previously (Jack et al. 1988). Digoxigenin-labeled DNA probes for detection of Dfd and Antp transcripts were prepared from pBstAntp digested with BamHIEcoRI and from pcDfd41 digested with HindIII-EcoRV (Regulski et al. 1987; McGinnis et al. 1990) using the Genius labeling kit from Boehringer Mannheim. The procedure of whole-mount in situ hybridization was based on the method of Tautz and Pfeifle (1989).

\section{Acknowledgments}

We are grateful to Nadine McGinnis for help with cuticular preparations, strain amplification, and antibody staining of transformant embryos, to Mike Kuziora and Scott Dessain for help initiating this project, and to Chaoyang Zeng for constructing one of the chimeric genes. We thank Cornelius Gross, Brad Jones, Acaimo Gonzáles-Reyes, Guy Vezina and Katherine Harding for reading and commenting on the manuscript. We are also indebted to Cornelius Gross for communicating unpublished results. This research was supported by a grant from the National Institutes of Health (PO1-GM39813).

The publication costs of this article were defrayed in part by payment of page charges. This article must therefore be hereby marked "advertisement" in accordance with 18 USC section 1734 solely to indicate this fact.

\section{References}

Affolter, M., A. Percival-Smith, M. Müller, W. Leupin, and W.J. Gehring. 1990. DNA binding properties of the purified Antennapedia homeodomain. Proc. Natl. Acad. Sci. 87: 40934097.

Beachy, P.A., M.A. Krasnow, E.R. Gavis, and D.S. Hogness. 1988. An Ultrabithorax protein binds sequences near its own and the Antennapedia P1 promoters. Cell 55: 1069-1081.

Bergson, C. and W. McGinnis. 1990. An autoregulatory enhancer element of the Drosophila homeotic gene Deformed. EMBO I. 9: 4287-4297.

Campos-Ortega, J.A. and V. Hartenstein. 1985. The embryonic development of Drosophila melanogaster. Springer-Verlag, Berlin, Germany.

Damante G. and R. Di Lauro. 1991. Several regions of Antennapedia and thyroid transcription factor 1 homeodomains contribute to DNA binding specificity. Proc. Natl. Acad. Sci. 88: 5388-5392.

Dessain S., C.T. Gross, M.A. Kuziora, and W. McGinnis. 1992. Antp-type homeodomains have distinct DNA-binding specificities that correlate with their different regulatory functions in embryos. EMBO /. 11: 991-1002.

Ekker, S.C., K.E. Young, D.P. von Kessler, and P.A. Beachy. 1991. Optimal DNA sequence recognition by the Ultrabithorax homeodomain of Drosophila. EMBO \%. 10: 11791186.

Florence, B., R. Handrow, and A. Laughon. 1991. DNA-binding specificity of the fushi tarazu homeodomain. Mol. Cell. Biol. 1991: 3613-3623.

Garcia-Bellido, A. 1977. Homeotic and atavic mutations in insects. Am. Zool. 17: 613-629.

Gehring, W.J. 1987. Homeoboxes in the study of development. Science 236: 1245-1252.

Gibson, G. and W.J. Gehring. 1988. Head and thoracic transformation caused by ectopic expression of Antennapedia during Drosophila development. Development 102: 657-675.

Gibson, G., A. Schier, P. LeMotte, and W.J. Gehring. 1990. The specificities of Sex combs reduced and Antennapedia are defined by a distinct portion of each protein that includes the homeodomain. Cell 62: 1087-1103.

González-Reyes, A. and G. Morata. 1990. The developmental effect of overexpressing a $U b x$ product in Drosophila embryos is dependent on its interactions with other homeotic products. Cell 61: 515-522.

Hafen, E., M. Levine, and W.J. Gehring. 1984. Regulation of Antennapedia transcript distribution by the bithorax complex in Drosophila. Nature 307: 287-289.

Hanes, S.D. and R. Brent. 1989. DNA specificity of the bicoid activator protein is determined by homeodomain recognition helix residue 9. Cell 57: 1275-1283.

- 1991. A genetic model for interaction of the homeodomain recognition helix with DNA. Science 251: 426430.

Hayashi, S. and M.P. Scott. 1990. What determines the specificity of action of Drosophila homeodomain proteins? Cell 63: $883-894$.

Ingraham, H.A., S.E. Flynn, J.W. Voss, V.R. Albert, M.S. Kapiloff, L. Wilson, and M.G. Rosenfeld. 1990. The POU-specific domain of Pit-1 is essential for sequence-specific, high affinity DNA binding and DNA-dependent Pit-1-Pit-1 interactions. Cell 61: 1021-1033. 
Jack, T., M. Regulski, and W. McGinnis, 1988. Pair-rule segmentation genes regulate the expression of the homeotic selector gene, Deformed. Genes \& Dev. 2: 635-651.

Jarvis, E.E., K.L. Clark, and G.F. Sprague, Jr. 1989. The yeast transcription activator PRTF, a homolog of the mammalian serum response factor, is encoded by the MCMl gene. Genes \& Dev. 3: 936-945.

Karess, R.E. and G.M. Rubin. 1984. Analysis of P-transposable element functions in Drosophila. Cell 38: 135-146.

Kaufman, T.C., M.A. Seeger, and G. Olsen. 1990. Molecular and genetic organization of the Antennapedia gene complex of Drosophila melanogaster. Adv. Genet. 27: 309-362.

Keleher, C.A., C. Goutte, and A.D. Johnson. 1988. The yeast cell-type-specific repressor alpha-2 acts cooperatively with a non-cell-type-specific protein. Cell 53: 927-936.

Kissinger, C.R., B. Liu, E. Martin-Blanco, T.B. Kornberg, and C.O. Pabo. 1990. Crystal structure of an engrailed home-

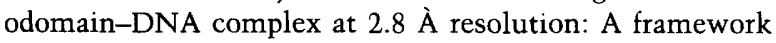
for understanding homeo domain-DNA interactions. Cell 63: $579-590$.

Kristie, T.M. and P.A. Sharp. 1990. Interactions of the Oct-1POU subdomains with specific DNA sequences and with the HSV $\alpha$-trans-activator protein. Genes \& Dev. 4: 23832396.

Kunkel, T.A. 1985. Rapid and efficient site-specific mutagenesis without phenotypic selection. Proc. Natl. Acad. Sci. 82: $488-492$.

Kuziora, M.A. and W. McGinnis. 1989. A homeodomain substitution changes the regulatory specificity of the Deformed protein in Drosophila embryos. Cell 59: 563-571.

- 1988. Autoregulation of a Drosophila homeotic selector gene. Cell 55: 477-485.

- 1991. Altering the target specificity of the Deformed protein in Drosophila embryos by substituting the Abdominal-B homeodomain. Mech. Dev. 33: 83-94.

Laughon, A. and M.P. Scott. 1984. Sequence of a Drosophila segmentation gene: Protein structure homology with DNAbinding proteins. Nature 310: 25-31.

Levine, M. and T. Hoey. 1988. Homeobox proteins as sequence specific transcription factors. Cell 55: 537-540.

Lewis, E.B. 1978. A gene complex controlling segmentation in Drosophila. Nature 276: 565-570.

Mann, R.S. and D.S. Hogness. 1990. Functional dissection of Ultrabithorax proteins in D. melanogaster. Cell 60: 597610.

McGinnis, N., M.A. Kuziora, and W. McGinnis. 1990. Human Hox-4.2 and Drosophila Deformed encode similar regulatory specificities in Drosophila embryos and larvae. Cell 63: 969976.

Mismer, D. and G.M. Rubin. 1987. Analysis of the promoter of the ninaE opsin gene in Drosophila melanogaster. Genetics 116: 565-578.

Müller, M., M. Affolter, W. Leupin, G. Otting, K. Wüthrich, and W.J. Gehring. 1988. Isolation and sequence-specific DNA binding of the Antennapedia homeodomain. EMBO $J$. 7: 4299-4304.

Otting, G., Y. Qian, M. Müller, M. Affolter, W. Gehring, and K. Wüthrich. 1988. Secondary structure determination for the Antennapedia homeodomain by nuclear magnetic resonance and evidence for a helix-turn-helix motif. EMBO I. 7: 43054309.

Otting, G., Y.Q. Qian, M. Billeter, M. Müller, M. Affolter, W.J. Gehring, and K. Wüthrich. 1990. Protein-DNA contacts in the structure of a homeodomain-DNA complex determined by nuclear magnetic resonance spectroscopy in solution. EMBO /. 9: 3085-3092.
Percival-Smith, A., M. Müller, M. Affolter, and W.J. Gehring. 1990. The interaction with DNA of wild-type and mutant fushi tarazu homeodomains. EMBO I. 9: 3967-3974.

Pirotta, V. 1988. Vectors for P-mediated transformations in Drosophila. In Vectors: A survey of molecular cloning vectors and their uses (ed. R.L. Rodriguez and D.T. Reinhardt), pp. 437-456. Butterworths, Boston, MA.

Qian, Y.Q., M. Billeter, G. Otting, M. Müller, W.J. Gehring, and K. Wüthrich. 1989. The structure of the Antennapedia homeodomain determined by NMR spectroscopy in solution: Comparison with prokaryotic repressors. Cell 59: 573-580.

Regulski, M., S. Dessain, N. McGinnis, and W. McGinnis. 1991. High-affinity binding sites for the Deformed protein are required for the function of an autoregulatory enhancer of the Deformed gene. Genes \& Dev. 5: 278-286.

Regulski, M., N. McGinnis, R. Chadwick, and W. McGinnis. 1987. Developmental and molecular analysis of Deformed: A homeotic gene controlling Drosophila head development. EMBO 1. 6: 767-777.

Rubin, G.M. and A.C. Spradling. 1982. Genetic transformation of Drosophila with transposable element vectors. Science 218: $348-353$.

Schneuwly, S., R. Klemenz, and W.J. Gehring. 1987. Redesigning the body plan of Drosophila by ectopic expression of the homoeotic gene Antennapedia. Nature 325: 816-818.

Scott, M.P., J.W. Tamkun, and G.W. Hartzell III. 1989. The structure and function of the homeo domain. Biochim. Biophys. Acta 989: 25-48.

Spradling, A.C. and G.M. Rubin. 1982. Transposition of cloned P-elements into Drosophila germ line chromosomes. Science 218: 341-347.

Stern, S., M. Tanaka, and W. Herr. 1989. The Oct-1 homeodomain directs formation of a multiprotein-DNA complex with the HSV transactivator VP16. Nature 341: 624-630.

Struhl, G. 1982. Genes controlling segmental specification in the Drosophila thorax. Proc. Natl. Acad. Sci. 79: 7380-7384.

Sturm, R.A. and W. Herr. 1988. The POU domain is a bipartite DNA-binding structure. Nature 336: 601-604.

Tautz, D. and C. Pfeifle. 1989. A non-radioactive in situ hybridization method for localization of specific RNAs in Drosophila embryos reveals translational control of the segmentation gene hunchback. Chromosoma 98: 81-85.

Treacy, M.N., X. He, and M.G. Rosenfeld. 1991. I-POU: A POUdomain protein that inhibits neuron-specific gene activation. Nature 350: 577-584.

Treisman, J., E. Harris, and C. Desplan. 1991. The paired box encodes a second DNA-binding domain in the Paired homeodomain protein. Genes \& Dev. 5: 594-604.

Treisman, J., P. Gonczy, M. Vashishtha, E. Harris, and C. Desplan. 1989. A single amino acid can determine the DNA binding specificity of homeodomain proteins. Cell 59: 553562.

Van der Meer, J.M. 1977. Optical clean and permanent whole mount preparation for phase contrast microscopy of cuticular structures of insect larvae. Dros. Inf. Serv. 52: 160.

Wolberger, C., A.K. Vershon, B. Liu, A.D. Johnson, and C.O. Pabo. 1991. Crystal structure of a MAT- $\alpha 2$ homeodomainoperator complex suggests a general model for homeodomain-DNA interactions. Cell 67: 517-528.

Zink, B., Y. Engstrom, W.J. Gehring, and R. Paro. 1991. Direct interaction of the Polycomb protein with Antennapedia regulatory sequences in polytene chromosomes of Drosophila melanogaster. EMBO \%. 10: 153-162. 


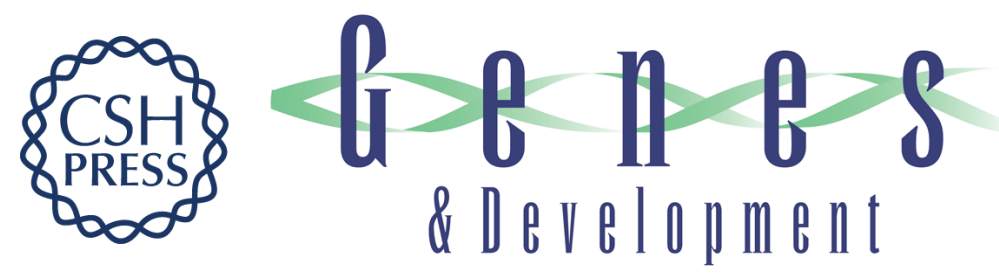

\section{Mapping functional specificity in the Dfd and Ubx homeo domains.}

L Lin and W McGinnis

Genes Dev. 1992, 6:

Access the most recent version at doi:10.1101/gad.6.6.1071 $\begin{array}{ll}\text { References } & \begin{array}{l}\text { This article cites } 55 \text { articles, } 15 \text { of which can be accessed free at: } \\ \text { http://genesdev.cshlp.org/content/6/6/1071.full.html\#ref-list-1 }\end{array}\end{array}$

License

Email Alerting Service

Receive free email alerts when new articles cite this article - sign up in the box at the top right corner of the article or click here.

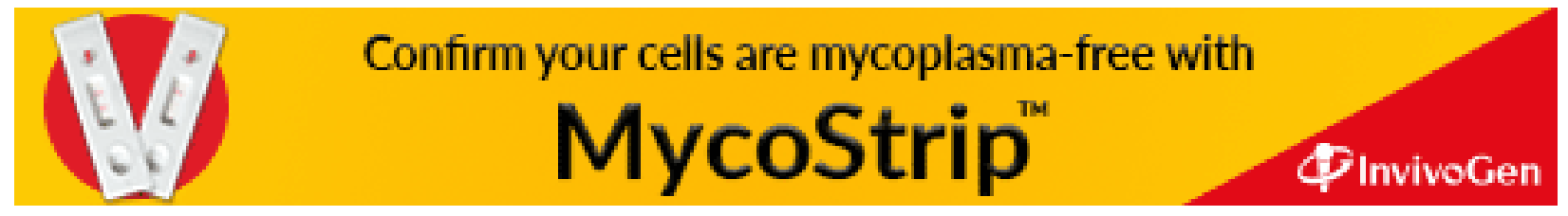

